\section{Neue Therapiestrategien bei CLL und malignen Lymphomen Überlebensverlängerung - Ferne Vision oder baldige Realität?}

Patienten mit chronischer lymphatischer Leukämie (CLL) und anderen Lymphomen können dank moderner Therapiestrategien auf eine deutliche Lebensverlängerung hoffen. Dafür sprechen neueste, auf einem von MedacSchering Onkologie unterstützten Symposium im Rahmen der Jahrestagung der DGHO 2004 vorgestellte Studienergebnisse.

Der Anti-CD52-Antikörper Alemtuzumab (MabCampath ${ }^{\circledR}$ ) induziert selbst bei Patienten mit therapie-refraktärer, stark vorbehandelter CLL langanhaltende Remissionen, und eliminiert bei ca. $20 \%$ der Patienten die Minimale Resterkrankung (MRD) aus Blut und Knochenmark, was mit einem verlängerten Überleben korreliert.

Hohe Ansprechraten auf MabCampath $^{\circledR}$ wurden z. B. in der CLL2HStudie der Deutschen CLL-Studiengruppe (DCLLSG) bei Patienten mit Fludarabin-refraktärer rezidivierter CLL erzielt, insbesondere auch bei denjenigen Patienten, die u. a. aufgrund eines unmutierten IgVH-Gens sowie chromosomaler Aberrationen eine besonders schlechte Prognose hatten: Eine komplette (CR) bzw. partielle Remission (PR) wurde bei 14 von 27 (52\%) der Patienten mit un- mutiertem IgVH-Gen erreicht, bei 5 von $13(38 \%)$ der Patienten mit 11q-Deletion sowie sogar bei 7 von 13 $(54 \%)$ der Patienten mit 17p-Deletion, berichtete PD Dr. Stephan Stilgenbauer vom Universitätsklinikum Ulm.

Weiter optimieren lässt sich die Therapie der CLL durch Kombination der synergistisch wirkenden Substanzen Alemtuzumab (MabCampath ${ }^{\circledR}$ ) und Fludarabin (Fludara ${ }^{\circledR}$ ), wie die Arbeitsgruppe um Prof. Dr. Andreas Engert, Universitätsklinikum Köln, in der FluCam-Studie bei 34 rezidivierten bzw. therapierefraktären CLLPatienten zeigen konnte. Auf die Kombination aus Alemtuzumab und Fludarabin sprachen 10 Patienten mit einer Komplettremission und 19 mit einer partiellen Remission bei einer Gesamtansprechrate von $85 \%$ an. $44 \%$ dieser Patienten wurden auch im peripheren Blut MRD-negativ.

Die Radioimmuntherapie mit Zevalin ${ }^{\circledR}$ stellt nach Ansicht von Experten ein attraktives, da hocheffizientes und sehr gut verträgliches Prinzip zur Behandlung vorbehandelter NonHodgkin-Lymphome dar. Dabei zerstört das mit dem Chelator Tiuxetan an den monoklonalen Anti-CD20-BZell-Antikörper Ibritumomab gekoppelte Radionuklid 90Yttrium (Ytracis $\left.^{\circledR}\right)$ hochspezifisch die strahlensensiblen Lymphomzellen auch in schwer zugänglichen Tumorbereichen unter Schonung des umliegenden Gewebes.
Die Radioimmuntherapie besticht vor allem dadurch, dass sie kurz, sicher, einfach durchführbar und auch bei älteren Patienten gut verträglich ist und hat schon jetzt einen hohen Stellenwert durch Induktion hoher CRRaten und lang anhaltender Remissionen auch im Rezidiv, erläuterte Prof. Dr. Lorenz Trümper vom Universitätsklinikum Göttingen.

Seit April 2004 steht Zevalin ${ }^{\circledR}$ für Patienten mit CD20-positivem follikulären B-Zell-Non-Hodgkin-Lymphom zur Verfügung, die nach einer Immuntherapie mit Rituximab rezidivieren oder refraktär sind. Geprüft wird die Wirksamkeit des Radioimmunkonjugats zurzeit auch bei anderen Lymphom-Entitäten. So sind Studien mit Zevalin ${ }^{\circledR}$ beim Mantelzell-Lymphom und diffus-großzelligen NHL auf dem Weg, um die an kleinen Fallzahlen beobachtete Wirksamkeit des Radioimmunkonjugats bei aggressiven NHL zu bestätigen. Untersucht wird darüber hinaus die Integration von Zevalin ${ }^{\circledR}$ in Konsolidierungsstudien und myeloablative Konzepte, bei denen eine RIT-Dosiseskalation in Kombination mit hochdosierten Chemotherapien und Stammzelltransplantation eingesetzt wird.

Weitere Informationen bei: MedacSchering Onkologie Dr. Karin Grassmann

Tel. +49 89 745144-0, Fax -40

E-mailk.grassmann@mso-pharma.de

\title{
PharmaTicker+++ PharmaTicker+++ PharmaTicker+++ PharmaTicker+++
}

Bristol-Myers Squibb GmbH. Seit Oktober 2004 werden sämtliche Medikamente der Geschäftseinheit HIV/AIDS mit fälschungssicheren Verpackungen ausgestattet. Die HIV-Medikamente sind mit dem von der november AG entwickelten DNA-Code versehen. Die Überprüfung erfolgt mit Hilfe eines Prüfstifts oder Handscanners und garantiert Großhandel und Apotheken die Echtheit des Präparates.

Weitere Informationen bei:

Pleon Healthcare $\mathrm{GmbH}$, Dr. Anke Helten

Tel. +49 89 59042-226, Fax -237

E-mail Anke.Helten@pleon.com
AstraZeneca GmbH. Die American Association of Clinical Oncology empfiehlt, Aromatasehemmer wie z.B. Anastrozol (Arimidex ${ }^{\circledR}$ ) in der adjuvanten Therapie des frühen Mammakarzinoms bei postmenopausalen Frauen einzusetzen, um das Rezidivrisiko zu reduzieren. Damit ist ein Paradigmenwechsel in der hormonellen Brustkrebstherapie vollzogen. Aromatasehemmer lösen den seit Jahrzehnten geltenden Goldstandard Tamoxifen ab.

Weitere Informationen bei

Publicis Vital PR, Anja Dörner

Tel. +49 221 912719-21, Fax -47

E-mail anja.doerner@publicis-vital-pr.de
Lilly Deutschland GmbH. In Deutschland ist das erste Krebsmedikament zur Behandlung des Asbesttumors auf dem Markt. Alimta ${ }^{\circledR}$ (Wirkstoff Pemetrexed), ein so genannter MultiTarget-Enzym-Inhibitor, wurde von Eli Lilly \& Co. mit der Universität von Princeton, NJ, USA, entwickelt und ist in der EU für die Behandlung des malignen Pleuramesothelioms (Asbesttumor) und zur Rezidiv-Therapie des nichtkleinzelligen Bronchialkarzinoms zugelassen

Weitere Informationen bei:

Lilly Deutschland $\mathrm{GmbH}$, Medizinische Information

Tel. +49 6172 273-2976

E-mail Med_Info@lilly.com 


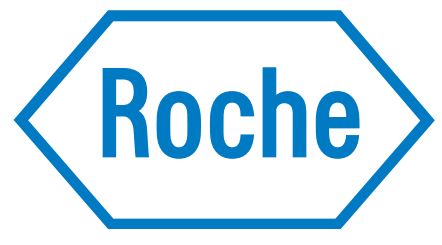

\section{Erlotinib erhält Zulassung in den USA für NSCLC \\ Targeted Therapy mit signifikanter Steigerung der Überlebensrate}

Die US-amerikanische Gesundheitsbehörde FDA hat den TyrosinkinaseHemmer Erlotinib (Tarceva ${ }^{\circledR}$ ) in einem beschleunigten Verfahren zur 2nd und 3rd Line-Therapie von Patienten mit lokal fortgeschrittenem oder metastasiertem, nicht-kleinzelligem Lungenkrebs (Non-Small Cell Lung Cancer, NSCLC) in den USA zugelassen. Erlotinib ist der erste und einzige gezielt auf den Epidermal Growth Factor Receptor (EGFR) gerichtete Wirkstoff, der bei Patienten mit NSCLC einen signifikanten Überlebensvorteil erzielt.

Die FDA-Zulassung basiert auf den Ergebnissen einer randomisierten, placebokontrollierten Phase-III-Studie (BR.21-Studie) an 731 Patienten mit fortgeschrittenem NSCLC, die zuvor mit ein bis zwei Chemotherapien vorbehandelt worden waren. Die mit Erlotinib behandelten Patienten überlebten signifikant länger, als die Patienten in der Placebogruppe (6,7 vs. 4,7 Monate, $\mathrm{p}<0,0001$ ), was einer Steigerung um $42 \%$ entspricht. Zudem ließ sich das progressionsfreie Überleben von 1,8 auf 2,2 Monate verlängern $(\mathrm{p}<0,0001)$. Die Überlebensrate konnte nach einem Jahr unter Verum um $45 \%$ verbessert werden.

«Erlotinib ist der erste Vertreter seiner Klasse, der bei Patienten mit fortgeschrittenem Lungenkrebs einen signifikanten Überlebensvorteil bringt», erklärte William M. Burns, Leiter der Division Pharma bei Roche. «Die Geschwindigkeit, mit der die FDA Erlotinib zugelassen hat, unterstreicht diese Tatsache. Wir freuen uns, dass Patienten in den USA jetzt von diesem innovativen Medikament profitieren können. Wir arbeiten eng mit der EMEA (European Medicines Evaluation Agency) und den Zulassungsbehörden anderer Länder zusammen, um das Medikament so rasch wie möglich auch weiteren $\mathrm{Pa}$ tienten zugänglich machen zu können.» Die Zulassungsgesuche für Erlotinib werden gegenwärtig geprüft.

\section{Erlotinib auch bei Pankreas- \\ Karzinom wirksam}

Analog zum signifikanten Überlebensvorteil bei Patienten mit NSCLC hat der Tyrosinkinase-Inhibitor auch in einer Phase-III-Studie bei Patienten mit lokal fortgeschrittenem oder metastasiertem Pankreas-Karzinom die Überlebensrate verbessert. Die Daten belegen eine Verlängerung der Gesamtüberlebenszeit, womit die Studie ihren primären Endpunkt erreicht hat.

\section{Neuer Wirkmechanismus EGFR-Hemmung}

Erlotinib ist ein «Small Molecule», das auf den humanen epidermalen Wachstumsfaktor-Rezeptor (HER 1) abzielt. HER1, auch EGFR genannt, ist eine wichtige Komponente des HER1-Signalwegs, der an der Entstehung und der Progression zahlreicher Krebsarten beteiligt ist. Erlotinib hemmt das Tumorzellwachstum, indem es durch Blockade der Tyrosinkinase-Aktivität innerhalb der Zelle die EGFR/HER1-vermittelte Signalübertragung verhindert.

\section{Referenz}

Shepherd F: A randomized placebo-controlled trial of erlotinib in patients with advanced nonsmall cell lung cancer (NSCLC) following failure of 1st line or 2nd line chemotherapy. A National Cancer Institute of Canada Clinical Trials Group (NCIC). ASCO 2004 (abstr 7022).

Weitere Informationen be: Dr. med. H.-U. Jelitto Hoffmann-La Roche AG Emil-Barell-Straße 1,

D-79639 Grenzach-Wyhlen

Tel. +49 7624 14-2400, Fax -3366 www.roche.de

\section{PharmaTicker+++ PharmaTicker+++ PharmaTicker+++ PharmaTicker+++}

MSD Sharp \& Dohme GmbH. Caspofungin ist als empirische Antimykotikatherapie bei Patienten mit Neutropenie und anhaltendem Fieber genauso wirksam, aber allgemein besser verträglich als liposomales Amphotericin B. Das zeigten die Ergebnisse der bislang größten Studie zu dieser Thematik, deren Ergebnisse kürzlich im New England Journal of Medicine veröffentlicht wurden.

Weitere Informationen bei:

MSD Sharp \& Dohme GmbH, Annette Menze

Tel. +49 89 45611353, Fax -29

E-mail annette menzel@msd.de
Gilead Sciences GmbH. Eine aktuelle Studie zur empirischen Therapie von Mykosen, die erstmals im Rahmen der gemeinsamen Jahrestagung von DGHO, ÖGHO, SGH und SGMO im Oktober 2004 in Innsbruck präsentiert wurde, zeigt, dass auch flexible, individuell an den Zustand und das Risiko des Patienten angepasstes Dosierungen von lipsomalem Amphotericin B (AmBisome $\left.{ }^{\circledR}\right)$ eine wirksame Behandlungsalternative darstellen.

Gilead Sciences GmbH, Dr. Sarita Chaubal

Tel. +4989 899890-12, Fax -49

E-mail sarita.chaubal@gilead.com essex pharma GmbH. Patientinnen mit rezidiviertem Ovarialkarzinom, die nicht mit Platinhaltigen Kombinationen behandelt werden können, profitieren von einer effektiven Monochemotherapie. So das Ergebnis einer randomisierten Phase-III-Studie, in der 474 Patientinnen entweder mit $50 \mathrm{mg} / \mathrm{m}^{2}$ pegyliertem liposomalem Doxorubicin alle 28 Tage oder mit $1,5 \mathrm{mg} / \mathrm{m}^{2}$ Topotecan täglich an 5 aufeinander folgenden Tagen alle 3 Wochen behandelt wurden. essex pharma GmbH, Dr. Detlef Hecker

Tel. +49 89 62731-277, Fax -253

E-mail detlef.hecker@essex.de 